\title{
Some Remarks on BRS Transformations, Anomalies and the Cohomology of the Lie Algebra of the Group of Gauge Transformations
}

\author{
L. Bonora ${ }^{\star, 1}$ and P. Cotta-Ramusino ${ }^{\star \star, 2}$ \\ 1 Center for Theoretical Physics, Laboratory for Nuclear Science and Department of Physics, \\ Massachusetts Institute of Technology, Cambridge, MA 02139, USA \\ 2 Lyman Laboratory of Physics, Harvard University, Cambridge, MA 02138, USA
}

\begin{abstract}
We show that ghosts in gauge theories can be interpreted as MaurerCartan forms in the infinite dimensional group $\mathscr{G}$ of gauge transformations. We examine the cohomology of the Lie algebra of $\mathscr{G}$ and identify the coboundary operator with the BRS operator. We describe the anomalous terms encountered in the renormalization of gauge theories (triangle anomalies) as elements of these cohomology groups.
\end{abstract}

\section{Introduction}

A few years ago Becchi et al. $[1,2]$ noticed that in gauge theories the effective Lagrangian (with gauge breaking and ghost terms included) is invariant under a special class of transformations (BRS transformations). The ensuing WardTakahashi identities allow an elegant proof of renormalizability and the nilpotent nature of the BRS transformations is apparently the key for the unitarity of the $S$-matrix.

More recently it was discovered [3] that many effective Lagrangians in gauge theories are invariant also under a second set of transformations, which we call anti-BRS transformations.

BRS transformations have attracted much interest ever since (see for instance [4] or [5]). There have been also some attempts to give a geometrical interpretation of the BRS transformations and the ghost fields. In [6] the ghost fields are viewed essentially as the "vertical part" of a connection form (locally defined). Reference [7] gives a modification of the ideas of [6] and attempts to give a geometrical interpretation of the antighosts as well. However, both attempts exhibit unsatisfactory aspects.

* Permanent address: Istituto di Física dell'Università di Padova and I.N.F.N. Sezione di Padova, Italy. Research supported in part by the U.S. Department of Energy (DOE) under contract DE-ACO276ER03069

$\star \star$ Permanent address: Istituto di Fisica dell'Università di Milano and I.N.F.N. Sezione di Milano, Italy. Research supported in part by C.N.R., I.N.F.N., and by the National Science Foundation under Grant PHY79-16812 
In the present work we do not try to improve the same interpretation, rather we change geometrical framework. Our main objects will be the space of connections and the group of gauge transformations acting on it. In this way we not only avoid the flaws of the previous interpretations, but construct a clear setting where the problem of Adler-Bell-Jackiw (ABJ) anomalies can be dealt with.

The paper is organized as follows:

In Sect. 2, we recall some definitions for BRS transformations and anomalies (Part A), and we fix some basic notations for the geometry of gauge fields (Part B).

In Sect. 3, we identify the ghost field as the Maurer-Cartan form on the group of gauge transformations; the anticommuting character of the ghosts is due to the fact that they are sections of an exterior algebra bundle. The BRS transformation on the space of vector potentials (connections) turns out to be a sort of symbolic representation of all the infinitesimal gauge transformations.

In Sect. 4 we consider the cohomology of the Lie algebra of the gauge groups with coefficients in the space of functionals on the space of connections. The coboundary operator for this cohomology can be viewed as the BRS operator. We find also some interesting connections with the geometry of gauge orbits: a functional with $p$-ghosts can be interpreted as a "vertical" $p$-form on the manifold of all connections (vector potentials).

In Sect. 5 we consider the "local" version of the cohomology discussed in Sect. 4 and we study the problem of finding the anomalies, which we identify with the elements of the "local" cohomology groups. For a previous study on the subject, see [10].

Sect. 6 contains a few comments.

\section{Some Basic Definitions and Notations}

\section{A) BRS Transformations and Anomalies}

The (infinitesimal) BRS transformations are usually written in the following way:

(a) $A_{\mu}^{a}(x) \rightarrow A_{\mu}^{a}(x)+\lambda \jmath\left(A_{\mu}^{a}(x)\right)=: A_{\mu}^{a}(x)+\lambda\left(D_{\mu} c(x)\right)^{a}$,

(b) $c^{a}(x) \rightarrow c^{a}(x)+\lambda s\left(c^{a}(x)\right)=: c^{a}(x)-\frac{1}{2} \lambda f^{a b d} c^{b}(x) c^{d}(x)$,

(c) $\bar{c}^{a}(x) \rightarrow \bar{c}^{a}(x)+\lambda s\left(\bar{c}^{a}(x)\right)=: \bar{c}^{a}(x)+\lambda B^{a}(x)$,

where $A_{\mu}^{a}(x), c^{a}(x), \bar{c}^{a}(x)$ are, respectively, the vector potential, the ghost and the antighost fields; the index $a$ labels a basis of the Lie algebra of the (compact) gauge group; $f^{a b d}$ are the structure constants of the gauge Lie algebra; the index $\mu$ labels the coordinates of the (Euclidean) space-time; $D_{\mu}$ is the covariant derivative in the direction $\mu ; B^{a}(x)$ is an auxiliary field and $\lambda$ is an anticommuting $x$-independent parameter, which is the conventional device used in field theory whenever a match between commuting and anticommuting objects is needed. We have

$$
\Im\left(D_{\mu} c(x)\right)^{a}=-\frac{1}{2}\left(D_{\mu}[c(x), c(x)]\right)^{a}+f^{a b d}\left(D_{\mu} c(x)\right)^{b} c^{d}(x)=0,
$$

that is $\jmath^{2}=0$ (nilpotency of the BRS operator). 
One usually defines also an action of $s$ on the matter fields (see [16]), but, though extending to them what we are going to say is rather straightforward, we will not consider matter fields in this paper.

An integrated anomalous term can be defined as a local functional $Q$ of the gauge fields, the ghosts and the matter fields, such that $s Q=0$ and there exists no functional $Q^{\prime}$ with $Q=s Q^{\prime}$ (see [16]). If at the quantum level we have an anomalous insertion (with ghost number one), then the Ward-Takahashi identities cannot be reproduced at higher order of the perturbative expansion and the theory is not renormalizable. Whether this insertion appears or not in the renormalization process is strictly dependent on the explicit form of the Lagrangian and will not be discussed here (see $[18,19])$. In this paper we are interested only in finding the possible anomalous terms and studying their geometrical meaning.

\section{B) Geometry of Yang-Mills Fields}

Here $M$ is an orientable, compact, connected Riemannian manifold (generally we take $S^{4}$ or $S^{3}$ ) and $G$ is a compact Lie group with an invariant inner product defined on its Lie algebra $\mathfrak{g}$. We note that $P(M, G)$ is a given $G$-principal bundle over $M$ and the following associated bundles are defined: $A d P=: P \times{ }_{G} G$ and ad $P$ $=: P \times{ }_{G} \mathfrak{g}$, where $G$ acts on itself (respectively on $\mathfrak{g}$ ) through the adjoint action. If $\mathfrak{g}^{*}$ is the dual of $\mathfrak{g}$ and we consider the coadjoint action of $G$ on $\mathfrak{g}^{*}$, we can also define the associated bundle ad* $P=P \times{ }_{G} \mathrm{~g}^{*}$.

For each bundle $B$, we denote the space of $C^{\infty}$-sections by $\Gamma(B)$. Let's define $\mathscr{G}=: \Gamma(\operatorname{Ad} P) ; E=\Gamma(\operatorname{ad} P) ; E^{*}=\Gamma\left(\operatorname{ad}^{*} P\right)$. Here $\mathscr{G}$ is a group under pointwise multiplication and is isomorphic to the group of automorphisms of $P ; E$ has a natural Lie algebra structure, induced by the Lie algebra structure in each fiber of $\operatorname{ad} P ; \mathscr{G}$ is called the group of gauge transformations; $E$ can be considered as the Lie algebra of $\mathscr{G}$ and $E^{*}$ as the dual of $E$ (see [11]).

Let us consider now the spaces [11]: $\Omega^{p}(M ; \operatorname{ad} P)=\Gamma\left(\bigwedge^{p} T^{*} M \otimes \operatorname{ad} P\right)$. Here $\Omega^{*}(M ; \operatorname{ad} P)=\sum_{p} \Omega^{p}(M ; \operatorname{ad} P)$ is the graded Lie algebra of forms with values in ad $P$ (locally, forms with values in $\mathfrak{g}$ ). The graded Lie algebra structure comes from the fact that combining the bracket operation in $g$ together with the exterior multiplication for forms on $M$, we have a map:

$$
[,]: \Omega^{p}(M ; \operatorname{ad} P) \times \Omega^{q}(M ; \operatorname{ad} P) \rightarrow \Omega^{p+q}(M ; \operatorname{ad} P),
$$

which satisfies the conditions:

$$
\begin{gathered}
{\left[\omega^{p}, \omega^{q}\right]=(-1)^{p q+1}\left[\omega^{q}, \omega^{p}\right],} \\
{\left[\omega^{p},\left[\omega^{q}, \omega^{r}\right]\right]=\left[\left[\omega^{p}, \omega^{q}\right], \omega^{r}\right]+(-1)^{p q}\left[\omega^{q},\left[\omega^{p}, \omega^{r}\right]\right],}
\end{gathered}
$$

where $\omega^{j} \in \Omega^{j}(M ; \operatorname{ad} P) \forall j$.

For any manifold $M$, we denote by $\Omega^{*}(M)=\sum_{p} \Omega^{p}(M)$ the algebra of differential forms. We can consider a mapping [11]:

$$
\bigwedge: \Omega^{q}(M ; \operatorname{ad} P) \times \Omega^{p}(M ; \operatorname{ad} P) \rightarrow \Omega^{p+q}(M) .
$$


Here $\bigwedge$ is defined by combining the exterior multiplication for forms on $M$ with the inner product on $\mathfrak{g}$. The Hodge operator is also defined in $\Omega^{*}(M ; \operatorname{ad} P)$ :

$$
*: \Omega^{q}(M ; \operatorname{ad} P) \rightarrow \Omega^{\operatorname{dim} M-q}(M ; \operatorname{ad} P),
$$

and an inner product in $\Omega^{*}(M ; \operatorname{ad} P)$ is:

$$
(\psi, \omega)=\int_{M} \psi \bigwedge^{*} \omega, \quad \psi, \omega \in \Omega^{*}(M ; \operatorname{ad} P)
$$

For $\psi, \omega \in \Omega^{1}(M ; \operatorname{ad} P)$, we have $[\psi, \omega] \in \Omega^{2}(M ; \operatorname{ad} P)$ and

$$
[\psi, \omega](X, Y)=[\psi(X), \omega(Y)]-[\psi(Y), \omega(X)]
$$

if $X, Y$ are vector fields on $M$. In particular,

$$
\frac{1}{2}[\psi, \psi](X, Y)=[\psi(X), \psi(Y)]
$$

Equations (2.8) and (2.9) hold of course also for any Lie algebra valued 1-forms on any manifold.

For each connection $A$ in $P(M, G)$, the covariant exterior derivative $d_{A}$ is a linear operator:

$$
d_{A}: \Omega^{q}(M ; \operatorname{ad} P) \rightarrow \Omega^{q+1}(M ; \operatorname{ad} P)
$$

which satisfies :

$$
d_{A}\left[\omega^{q}, \chi\right]=\left[d_{A} \omega^{q}, \chi\right]+(-1)^{q}\left[\omega^{q}, d_{A} \chi\right],
$$

$d\left(\omega^{q} \wedge \chi\right)=d_{A} \omega^{q} \wedge \chi+(-1)^{q} \omega^{q} \wedge d_{A} \chi, \quad \forall \omega^{q} \in \Omega^{q}(M ; \operatorname{ad} P), \quad \forall \chi \in \Omega^{*}(M ; \operatorname{ad} P)$

The inner product (2.7) allows us to define an operator $d_{A}^{*}: \Omega^{q}(M ; \operatorname{ad} P) \rightarrow \Omega^{q-1}(M ; \operatorname{ad} P)$ adjoint to $d_{A}$. The space of all connections on $P(M, G)$ is an affine space $\mathscr{A}$ modelled on $\Omega^{1}(M ; \operatorname{ad} P)[11]$. Thus $\mathscr{G}$ acts as a transformation group on $\mathscr{A}$. It is now possible to consider $\mathscr{G} ; E ; E^{*} ; \Omega^{q}(M ; \operatorname{ad} P)$ and consequently also $\mathscr{A}$ as Hilbert manifolds [13-15].

We choose $k>\frac{\operatorname{dim} M}{2}+1$ and consider the completions of the various spaces of sections with respect to the $k$-Sobolev norms. Let us denote these completions $\mathscr{G}_{k}{ }^{1} ; E_{k} ; E_{k}^{*} ; \Omega_{k}^{q}(M ; \operatorname{ad} P)$. Here $\mathscr{A}_{k}$ is the space of $k$-Sobolev connections and is an affine space modelled on $\Omega_{k}^{1}(M ; \operatorname{ad} P)$. Note that $\mathscr{G}_{k+1}$ acts on $\mathscr{A}_{k}$ with a $C^{\infty}$-action which is not generally free. To get a free action one must consider the space $\overline{\mathscr{A}}_{k}$ of irreducible connections in $\mathscr{A}_{k}$ and the group $\overline{\mathscr{G}}_{k+1}=: \frac{\mathscr{G}_{k+1}}{\text { center of } \mathscr{G}_{k+1}}$. Then

$$
\mathscr{P}_{k}=: \overline{\mathscr{A}}_{k}\left(\overline{\mathscr{A}}_{k} / \overline{\mathscr{G}}_{k+1} ; \overline{\mathscr{G}}_{k+1}\right)
$$

is a principal fibration $[12-15]$.

1 For the definition of $\mathscr{G}_{k}$ as a closed subset of a $k$-Sobolev completion of the space of sections in a suitable vector bundle, see $[13,14]$ 
The Lie algebra of $\overline{\mathscr{G}}_{k+1}$ is $E_{k+1}, \overline{\mathscr{A}}_{k}$ is a Hilbert manifold modelled on $\Omega_{k}^{1}(M ; \operatorname{ad} P)$.

In the principal bundle (2.13) the vertical space in $A \in \overline{\mathscr{A}}_{k}$ is given by [12-15]:

$$
V_{A}=\left\{\tau \in \Omega_{k}^{1}(M ; \operatorname{ad} P) \mid \exists \xi \in E_{k+1}, \text { such that } \tau=d_{A} \xi\right\} .
$$

A connection form $\alpha$ for $\mathscr{P}_{k}$ is given by:

with curvature form $[12,20]$ :

$$
\begin{aligned}
\alpha_{A}: T_{A} \tilde{\mathscr{A}}_{k} & \rightarrow E_{k+1}, \\
\tau & \rightsquigarrow\left(d_{A}^{*} d_{A}\right)^{-1} d_{A}^{*} \tau,
\end{aligned}
$$

$$
\begin{aligned}
K_{A}: T_{A} \tilde{\mathscr{A}}_{k} \times T_{A} \overline{\mathscr{A}}_{k} & \rightarrow E_{k+1}, \\
\tau_{1}, \tau_{2} & \rightsquigarrow\left(d_{A}^{*} d_{A}\right)^{-1}\left(b_{\tau_{1}^{h}}^{*}\left(\tau_{2}^{h}\right)-b_{\tau_{2}^{h}}^{*}\left(\tau_{1}^{h}\right)\right),
\end{aligned}
$$

where $\tau_{i}^{h}$ is the horizontal component of $\tau_{i}, b_{\tau_{i}^{h}}=:\left[\tau_{i}^{h}, \cdot\right], i=1,2$ and $b_{\tau_{i}^{h}}^{*}$ is the adjoint of $b_{\tau_{t}}$. Notice that $d_{A}$ and $d_{A}^{*}$ are continuous operators in the Sobolevnorms.

We assume from now on that all our $\infty$-dimensional spaces of sections have been completed with respect to a suitable Sobolev-norm. We will nevertheless omit the Sobolev indexes in $\mathscr{G}_{k+1}, \overline{\mathscr{G}}_{k+1}, \mathscr{A}_{k}, \overline{\mathscr{A}}_{k}, E_{k+1}, E_{k+1}^{*}, \Omega_{k}^{*}(M$, ad $P)$ to avoid a cumbersome notation.

\section{Ghost and BRS Transformations}

We want to give a representation of the objects and operations used in field theory which have been introduced in the first part of the previous section, by means of the differential geometric formalism presented in the second part. To this end we observe that the most natural way to interpret the transformation (2.1)(a) is regarding it as an infinitesimal transformation in the space of connections. Therefore the argument of the covariant derivative in (2.1) (a) multiplied by $\lambda$ must be an element of $\Omega^{0}(M ; \operatorname{ad} P)=E$. Now, if we remember that the ghost field in (2.1) (a) is an anticommuting object, it is natural to think of it as a differential form with values in E. Moreover Eq. (2.1) (b) shows that the s-transformation on the ghost satisfies an equation of the Maurer-Cartan type. Therefore, we identify the ghost field with the Maurer-Cartan form $\omega$ on the group $\mathscr{G}$.

Here $\omega$ is defined as the left invariant $E$-valued 1-form on $\mathscr{G}$, such that:

$$
\omega(\xi)=\xi^{*},
$$

where $\xi$ is a left invariant vector field on $\mathscr{G}$ and $\xi^{*}$ is the element of the Lie algebra $E$ corresponding to $\xi$. For simplicity, in the following we identify the elements of $E$ with the corresponding left invariant vector fields on $\mathscr{G}$.

For each connection $A$ and for each $\xi \in E: d_{A} \omega(\xi)=d_{A} \xi \in \Omega^{1}(M ; \operatorname{ad} P)$ and $A+d_{A} \omega(\xi)$ is another connection. We now call $d_{A} \omega$ a left invariant 1 -form on $\mathscr{G}$ with values in $\Omega^{1}(M ; \operatorname{ad} P)$, defined by:

$$
\begin{gathered}
d_{A} \omega: E \rightarrow \Omega^{1}(M, \operatorname{ad} P), \\
\xi \rightsquigarrow d_{A} \omega(\xi) .
\end{gathered}
$$


In other words if the action of $\mathscr{G}$ on $\mathscr{A}$ is denoted by $(A, g) \leadsto A \cdot g, A \in \mathscr{A}, g \in \mathscr{G}$ and $\varrho_{A}: \mathscr{G} \rightarrow \mathscr{A}$ is given by: $\varrho_{A}(g)=A \cdot g$, then $d_{A} \omega$ is the derivative of $\varrho_{A}$ at $g=$ identity. The transformation (2.1) (a) can now be rewritten in the following symbolic form:

$$
A \rightarrow A+d_{A} \omega .
$$

Expression (3.3) represents in a compact form all the possible infinitesimal gauge transformations, each of which is characterized by an element of the Lie algebra $E$; that is (3.3) stands for:

$$
(A, \xi) \rightarrow A+d_{A} \omega(\xi), \quad \xi \in E, A \in \mathscr{A} .
$$

Let us apply the transformation $\left(3.3^{\prime}\right)$ twice, antisymmetrizing with respect to the vector fields $\xi, \zeta \in E$ involved

$$
\begin{aligned}
(A, \xi, \zeta) & \rightarrow A+d_{A} \omega(\xi)+d_{A+d_{A} \omega(\zeta)} \omega(\zeta)-d_{A} \omega(\zeta)-d_{A+d_{A} \omega(\zeta)} \omega(\xi) \\
& =A+d_{A}[\omega(\xi), \omega(\zeta)] .
\end{aligned}
$$

Let's define an $\Omega^{1}(M$, ad $P)$-valued left invariant two-form on $\mathscr{G}$ by:

$$
\left[d_{A} \omega, \omega\right](\xi, \zeta):=\left[d_{A} \omega(\xi), \omega(\zeta)\right]+\left[\omega(\xi), d_{A} \omega(\zeta)\right], \quad \xi, \zeta \in E .
$$

Notice that we are in fact dealing with forms on $\mathscr{G}$, valued in the graded Lie algebra $\Omega^{*}(M$, ad $P)$; in this sense the definition (3.5) is nothing but an adaptation of Eq. (2.8). We have then

$$
\left[d_{A} \omega, \omega\right]-\frac{1}{2} d_{A}[\omega, \omega]=0,
$$

where the 2-form $[\omega, \omega]$ is such that $\frac{1}{2}[\omega, \omega](\xi, \zeta)=[\omega(\xi), \omega(\zeta)], \forall \xi, \zeta \in E$. Equation (3.6) is the same as Eq. (2.2); adding to the result of the transformation (3.4) the term: $-\frac{1}{2} d_{A}[\omega, \omega](\xi, \zeta)$, we get back to the connection $A$ where we started from (this corresponds to the nilpotent nature of the BRS transformations); the term $-\frac{1}{2} d_{A}[\omega, \omega](\xi, \zeta)$ is the exterior derivative of $\omega$, applied to the vector fields $\xi$ and $\zeta$; Eq. (2.1)(b) is the Maurer-Cartan equation for $\omega$, provided that we identify the BRS transformation on ghosts with the exterior differentiation for forms on $\mathscr{G}$. (For a short discussion about the anti-BRS transformations, see the appendix.)

From what we have seen in this section, the relevant group in studying the BRS invariance is the group of gauge transformations acting on the manifold of connections. Whenever we have an action of a group on a manifold we have natural way a cohomology of the Lie algebra of the group with coefficients in the algebra of differential forms on the manifold (see e.g. [21]). In our case the coboundary operator is exactly the BRS operator, as we shall see in the next section.

\section{Cohomology of the Lie Algebra of the Group of Gauge Transformations}

For each element $\xi$ in the Lie algebra $E$ of the group $\mathscr{G}$, we have a fundamental vector field $Z_{\xi}$ on the manifold of connections. Here $Z_{\xi}$ can be defined by its action 
on the functions in $\Omega^{0}(\mathscr{A})$; that is if $f: \mathscr{A} \rightarrow \mathbb{R}, A \in \mathscr{A}$ and exp is the exponential mapping in $\mathscr{G}$ :

$$
\left(Z_{\xi} f\right)(A)=\left.\frac{d}{d t} f(A \cdot \exp t \xi)\right|_{t=0}=\left.\frac{d}{d t} f\left(A+t d_{A} \xi\right)\right|_{t=0} .
$$

Obviously we have $Z_{\xi}(A)=d_{A} \omega(\xi)$, where $\omega$ is the Maurer-Cartan form. So $Z_{\xi}$ is generated by the transformation $\left(3.3^{\prime}\right)$.

Let $\theta_{\mathscr{A}}(\xi)$ be the Lie derivative along $Z_{\xi}$ :

$$
\theta_{\mathscr{A}}(\xi): \Omega^{p}(\mathscr{A}) \rightarrow \Omega^{p}(\mathscr{A}) .
$$

Since we are interested in functionals on the space of connections and not in $p$-forms with $p \geqq 1$, we will limit ourselves to considering the action of the operator $\theta_{\mathscr{A}}(\xi)$ on $\Omega^{0}(\mathscr{A})$. We define $\Gamma^{p}(E, \mathscr{A})$ as the space of $p$-linear skew mappings from $\underbrace{E \times \ldots \times E}_{p \text { times }}$ to $\Omega^{0}(\mathscr{A})$, which satisfy the following continuity condition: if $\psi \in \Gamma^{p}(E, \mathscr{A})$ and $A \in \mathscr{A}$, then the map $\psi_{A}$ given by:

$$
\begin{aligned}
\psi_{A}: & E \times \ldots \times E \rightarrow \mathbb{R}, \\
& \left(\xi_{1}, \ldots, \xi_{p}\right) \rightsquigarrow \psi\left(\xi_{1}, \ldots, \xi_{p}\right)(A)
\end{aligned}
$$

is continuous $\forall A \in \mathscr{A}$.

In the complex $\Gamma^{*}(E, \mathscr{A})=\sum_{p} \Gamma^{p}(E, \mathscr{A})$ we define an operator $\delta$ by:

$\delta: \Gamma^{p}(E, \mathscr{A}) \rightarrow \Gamma^{p+1}(E, \mathscr{A})$,

$$
\begin{aligned}
(\delta \psi)\left(\xi_{1}, \ldots, \xi_{p+1}\right)= & : \sum_{i=1}^{p+1}(-1)^{i+1} \theta_{\mathscr{A}}\left(\xi_{i}\right)\left(\psi\left(\xi_{1}, \ldots, \hat{\xi}_{i}, \ldots, \xi_{p+1}\right)\right) \\
& +\sum_{i<j}(-1)^{i+j} \psi\left(\left[\xi_{i}, \xi_{j}\right], \xi_{1}, \ldots, \hat{\xi}_{i}, \ldots, \hat{\xi}_{j}, \ldots, \xi_{p+1}\right),
\end{aligned}
$$

where ${ }^{\wedge}$ indicates omission.

Theorem 4.1. $\delta^{2}=0$, that is, $\delta$ is a coboundary operator.

The proof is completely analogous to proving that the square of the exterior derivative is zero for differential forms on a manifold. We define also, $\forall \xi \in E$ :

$$
\begin{aligned}
\theta_{E}(\xi): \Gamma^{p}(E, \mathscr{A}) & \rightarrow \Gamma^{p}(E, \mathscr{A}), \\
\left(\theta_{E}(\xi) \psi\right)\left(\xi_{1}, \ldots, \xi_{p}\right) & =\sum_{i}(-1)^{i} \psi\left(\left[\xi_{,}, \xi_{i}\right], \xi_{1}, \ldots, \hat{\xi}_{i}, \ldots, \xi_{p}\right), \\
i(\xi): \Gamma^{p}(E, \mathscr{A}) & \rightarrow \Gamma^{p-1}(E, \mathscr{A}), \\
(i(\xi) \psi)\left(\xi_{1}, \ldots, \xi_{p-1}\right) & =\psi\left(\xi, \xi_{1}, \ldots, \xi_{p-1}\right) . \\
\theta(\xi): \Gamma^{p}(E, \mathscr{A}) & \rightarrow \Gamma^{p}(E, \mathscr{A}), \\
(\theta(\xi) \psi)\left(\xi_{1}, \ldots, \xi_{p}\right) & =\left(\left(\theta_{\mathscr{A}}(\xi)+\theta_{E}(\xi)\right) \psi\right)\left(\xi_{1}, \ldots, \xi_{p}\right) .
\end{aligned}
$$


Theorem 4.2. The following identity holds $\forall \xi \in E$,

$$
\theta(\xi)=i(\xi) \delta+\delta i(\xi)
$$

The proof is analogous to the proof of a similar theorem for differential forms on a manifold if $\delta$ plays the role of the exterior derivative, $\theta(\cdot)$ the role of the Lie derivative along a vector field, $i(\cdot)$ the role of the contraction operator by a vector field.

The cohomology space of the complex $\left(\Gamma^{*}(E, \mathscr{A}), \delta\right)$ is called cohomology of $E$ with coefficients in $\Omega^{0}(\mathscr{A})$, and is denoted by $H^{*}(E, \mathscr{A})$. Note that $\forall p, H^{p}(E, \mathscr{A})$ is a $H^{0}(E, \mathscr{A})$-module. As for the "physical" meaning of our objects, notice that functionals of the gauge vector potentials with $p$ ghosts are cochains in $\Gamma^{p}(E, \mathscr{A})$, gauge invariant functionals with no ghosts are elements in $H^{0}(E, \mathscr{A}), \delta$ is the BRS operator, and (integrated) anomalous terms with $p$ ghosts are elements of $H^{p}(E, \mathscr{A})$.

The computation of $H^{*}(E, \mathscr{A})$ is in general difficult and probably not very interesting from the point of view of gauge theories. In fact in field theory we do not need generic $C^{\infty}$-functionals on the space of connections, but only functionals which are integrals over the base space $M$ of polynomials of the vector potentials, the ghosts and their derivatives. This is the reason why in the next sections we will consider the "local" cohomology of $E$.

We would like now to say a few words about the relation between the complex $\left(\Gamma^{*}(E, \mathscr{A}), \delta\right)$ and the geometry of gauge orbits. Let us consider the space $\overline{\mathscr{A}}$ of irreducible connections and the complex $\Gamma^{*}(E, \bar{A})$.

Definition. A differential form $\phi \in \Omega^{p}(\overline{\mathscr{A}}), \quad p \geqq 1$, is called vertical if $\phi\left(A ; \tau_{1}, \ldots, \tau_{p}\right)=0$ whenever one of the tangent vectors $\tau_{i}$ is horizontal.

Theorem 4.3. The space of vertical p-forms $(p \geqq 1)$ on $\overline{\mathscr{A}}$ is isomorphic to $\Gamma^{p}(E, \bar{A})$.

Proof. Let $\alpha$ be the connection form $(2.15), \alpha_{A}: T_{A} \overline{\mathscr{A}} \rightarrow E$ and $\forall \psi \in \Gamma^{p}(E ; \overline{\mathscr{A}})$ define the $p$-form $\hat{\psi}$ by:

$$
\hat{\psi}\left(A ; \tau_{1}, \ldots, \tau_{p}\right)=: \psi\left(\alpha_{A}\left(\tau_{1}\right), \ldots, \alpha_{A}\left(\tau_{p}\right)\right)(A), \quad \tau_{i} \in T_{A} \overline{\mathscr{A}}, A \in \overline{\mathscr{A}} .
$$

If $\tau_{i}$ is horizontal for some $i$, then $\alpha_{A}\left(\tau_{i}\right)=0$ and so $\hat{\psi}$ is a vertical $p$-form. Viceversa if $\phi$ is a vertical $p$-form, define $\tilde{\phi} \in \Gamma^{p}(E, \bar{A})$ by :

$$
\tilde{\phi}\left(\xi_{1}, \ldots, \xi_{p}\right)(A)=: \phi\left(A ; d_{A} \xi_{1}, \ldots, d_{A} \xi_{p}\right), \quad A \in \overline{\mathscr{A}}, \xi_{i} \in E
$$

Obviously $\phi=\hat{\tilde{\phi}}$ and the correspondence $\phi \rightarrow \tilde{\phi}$ is linear.

We now want to show that in the correspondence between $\Gamma^{*}(E, \overline{\mathscr{A}})$ and the space of vertical forms, the operator $\delta$, that is, the BRS operator, becomes a sort of "vertical" exterior derivative. Let us call $\bar{\delta}$ the exterior derivative in $\Omega^{*}(\overline{\mathscr{A}})$. Here $\forall \phi \in \Omega^{p}(\overline{\mathscr{A}})$, the vertical $p$-form $\phi_{V}$ is defined by:

$$
\phi_{V}\left(A ; \tau_{1}, \ldots, \tau_{p}\right)=: \phi\left(A ; d_{A} \alpha_{A}\left(\tau_{1}\right), \ldots, d_{A} \alpha_{A}\left(\tau_{p}\right)\right) .
$$


We have

Theorem 4.4. The notations being the same as before, the following identity holds:

$$
(\delta \psi)\left(\xi_{1}, \ldots, \xi_{p+1}\right)=(\bar{\delta} \hat{\psi})_{V}\left(A ; \tau_{1}, \ldots, \tau_{p+1}\right),
$$

where $\psi \in \Gamma^{p}(E, \overline{\mathscr{A}}) ; \xi_{i} \in E ; \tau_{i}$ are vector fields on $\overline{\mathscr{A}}$ such that $\xi_{i}=\alpha_{A}\left(\tau_{i}\right)$.

Proof.

$$
\begin{aligned}
& (\bar{\delta} \hat{\psi})\left(A ; \tau_{1}, \ldots, \tau_{p+1}\right)=\sum_{i=1}^{p+1} \mathscr{L}_{\tau_{i}}\left(\psi\left(\alpha_{A}\left(\tau_{1}\right), \ldots, \widehat{\alpha_{A}\left(\tau_{i}\right)}, \ldots, \alpha_{A}\left(\tau_{p+1}\right)\right)\right. \\
& \quad+\sum_{i<j}(-1)^{i+j} \psi\left(\alpha_{A}\left(\left[\tau_{i}, \tau_{j}\right]_{\mathscr{A}}\right), \alpha_{A}\left(\tau_{1}\right), \ldots, \widehat{\alpha_{A}\left(\tau_{i}\right)}, \ldots, \widehat{\alpha_{A}\left(\tau_{j}\right)}, \ldots, \alpha_{A}\left(\tau_{p+1}\right),\right.
\end{aligned}
$$

where $\mathscr{L}_{\tau_{i}}$ is the Lie derivative along $\tau_{i}$ and $[,]_{\bar{A}}$ is the bracket for vector fields in $\overline{\mathscr{A}}$. Now let $\tau_{i}=d_{A} \alpha_{A}\left(\tau_{i}\right)+\tau_{i}^{h}$, where $\tau_{i}^{h}$ is the horizontal component of $\tau_{i}$

by definition.

$$
\begin{aligned}
& \mathscr{L}_{d_{A} \alpha_{A}\left(\tau_{i}\right)}\left(\psi\left(\alpha_{A}\left(\tau_{1}\right), \ldots, \widehat{\alpha_{A}\left(\tau_{i}\right)}, \ldots, \alpha_{A}\left(\tau_{p+1}\right)\right)\right. \\
& \quad=\theta_{A}\left(\alpha_{A}\left(\tau_{i}\right)\right)\left(\psi\left(\alpha_{A}\left(\tau_{1}\right), \ldots, \widehat{\alpha_{A}\left(\tau_{i}\right)}, \ldots, \alpha_{A}\left(\tau_{p+1}\right)\right)\right),
\end{aligned}
$$

We have also

$$
\begin{aligned}
\alpha_{A}\left(\left[\tau_{i}, \tau_{j}\right]_{\mathscr{A}}\right) & =\alpha_{A}\left(\left[\tau_{i}^{h}+d_{A} \alpha_{A}\left(\tau_{i}\right), \tau_{j}^{h}+d_{A} \alpha_{A}\left(\tau_{j}\right)\right]_{\mathscr{A}}\right) \\
& =\alpha_{A}\left[d_{A} \alpha_{A}\left(\tau_{i}\right), d_{A} \alpha_{A}\left(\tau_{j}\right)\right]_{\mathscr{A}}-K_{A}\left(\tau_{i}^{h}, \tau_{j}^{h}\right) \\
& =\left[\alpha_{A}\left(\tau_{i}\right), \alpha_{A}\left(\tau_{j}\right)\right]-K_{A}\left(\tau_{i}, \tau_{j}\right),
\end{aligned}
$$

where $K_{A}$ is the curvature (2.16). Notice that we have used the structure equation for the curvature and the fact that the bracket of two fundamental vector fields corresponding to two elements of $E$, is equal to the fundamental vector field corresponding to the bracket of the two elements of $E$. This completes the proof.

\section{Local Cohomology and Anomalies}

As we mentioned before, the space $\Gamma^{p}(E, \mathscr{A})$ is too large; in field theory we need functionals which are integrals of polynomials in the fields, in the ghosts and in their derivatives. This is the reason why we want to define the "local" cohomology.

Let us consider a generic vector bundle $B$ : a $p$-linear mapping $L$ from $\Gamma(B) \times \ldots \times \Gamma(B)$ to $\Omega^{k}(M)$ is said to be "local" if:

$p$ times

$$
\operatorname{supp} L\left(\xi_{1}, \ldots, \xi_{p}\right) \subset \operatorname{supp} \xi_{1} \cap \operatorname{supp} \xi_{2} \cap \ldots \cap \operatorname{supp} \xi_{p}, \quad \xi_{i} \in \Gamma(B) .
$$

A theorem by Peetre [22] guarantees that, locally, $L$ is a differential operator.

Let us consider symmetric $m$-linear local maps $L_{m}$ from

$$
\underbrace{\Omega^{1}(M ; \operatorname{ad} P) \times \ldots \times \Omega^{1}(M ; \operatorname{ad} P)}
$$

$m$ times 
to $\Omega^{k}(M)$; fix $A_{0} \in \mathscr{A}$ and set $\eta(A)=: A-A_{0}, A \in \mathscr{A}$. A generic $k$-form dependent "locally" on the connection is an object such as

$$
L(A)=\sum_{m=0}^{s} L_{m}(\eta(A), \eta(A), \ldots, \eta(A))
$$

Let us call $D^{k}\left(\mathscr{A}, A_{0}, M\right)$ the set of all $k$-forms on $M$, depending on $A$ in the way explained before, with a finite number of $L_{m}$ 's. In local coordinates $\psi \in D^{k}\left(\mathscr{A}, A_{0}, M\right)$ is a $k$-form whose components are polynomials in $\eta(A)$ and its derivatives. We consider now $p$-cochains from $E \times \ldots \times E$ to $D^{k}\left(\mathscr{A}, A_{0}, M\right)$ with the property that, if $\phi$ is such a cochain, then

$$
p \text { times }
$$

$$
\operatorname{supp} \phi\left(\xi_{1}, \ldots, \xi_{p}\right)(A)\left(\operatorname{supp} \xi_{1} \cap \operatorname{supp} \xi_{2} \cap \ldots \cap \operatorname{supp} \xi_{p}, \quad \forall A \in \mathscr{A} .\right.
$$

We denote the space of such $p$-cochains by $S^{p, k}$ and set $S=: \sum_{p, k} S^{p, k}$.

Now it is clear that for $\psi \in D^{k}\left(\mathscr{A}, A_{0}, M\right)$ we have: $d \psi \in D^{k+1}\left(\mathscr{A}, A_{0}, M\right)$, where $(d \psi)(A)=: d \psi(A)$ and $d$ is the ordinary exterior derivative. If $\phi \in S^{p, k}$, define

$$
(d \phi)\left(\xi_{1}, \ldots, \xi_{p}\right)=: d\left(\phi\left(\xi_{1}, \ldots, \xi_{p}\right)\right)
$$

for $\xi_{i} \in E$. So $d: S^{p, k} \rightarrow S^{p, k+1}$ and $d^{2}=0$. But in $S$ we can define another differential operator in the following way: $\forall \psi \in D^{k}\left(\mathscr{A}, A_{0}, M\right)$ and $\forall \xi \in E$ set:

$$
\left[\theta_{\mathrm{loc}}(\xi) \psi\right](A)=:\left.\frac{d}{d t} \psi\left(A+t d_{A} \xi\right)\right|_{t=0}
$$

We have $d \theta_{\text {loc }}(\xi)=\theta_{\text {loc }}(\xi) d, \forall \xi \in E$. Let us define now

$$
\begin{aligned}
\delta_{\mathrm{loc}}: S^{p, k} \rightarrow & S^{p+1, k} \\
\left(\delta_{\mathrm{loc}} \phi_{p}^{k}\right)\left(\xi_{1}, \ldots, \xi_{p+1}\right)= & : \sum_{i=1}^{p}(-1)^{i+1} \theta_{\mathrm{loc}}\left(\xi_{i}\right)\left(\phi_{p}^{k}\left(\xi_{1}, \ldots, \hat{\xi}_{i}, \ldots, \xi_{p+1}\right)\right) \\
& +\sum_{i<j}(-1)^{i+j} \phi_{p}^{k}\left(\left[\xi_{i}, \xi_{j}\right], \xi_{1}, \ldots, \hat{\xi}_{i}, \ldots, \hat{\xi}_{j}, \ldots, \xi_{p+1}\right) .
\end{aligned}
$$

Of course $d \delta_{\text {loc }}=\delta_{\text {loc }} d$.

In the following, let $n$ be the dimension of $M$. Since $\forall \phi_{p}^{n} \in S^{p, n}$, we have $\int_{M} \phi_{p}^{n} \in \Gamma^{p}(E, \mathscr{A})$ and

$$
\int_{M} \delta_{\mathrm{loc}} \phi_{p}^{n}=\delta \int_{M} \phi_{p}^{n}
$$

Setting $\hat{d} \phi_{p}^{k}=:(-1)^{p} d \phi_{p}^{k}$ we have: $\delta \int_{M} \phi_{p}^{n}=0 \Leftrightarrow \exists \phi_{p+1}^{n-1}$ such that $\delta_{\text {loc }} \phi_{p}^{n}$ $+\hat{d} \phi_{p+1}^{n-1}=0$ and $\int_{M} \phi_{p}^{n}=\delta \int_{M} \phi_{p-1}^{n} \Leftrightarrow \exists \phi_{p}^{n-1}$ such that $\phi_{p}^{n}=\delta_{\mathrm{loc}} \phi_{p-1}^{n}+\hat{d} \phi_{p}^{n-1}$, that is, $\int_{M} \phi_{p}^{n} \in H^{p}(E, \mathscr{A}) \Leftrightarrow \phi_{p}^{n} \in H^{\delta_{\mathrm{loc}}} H^{d}\left(S^{p, n}\right)$, where $H^{\delta_{\mathrm{loc}}}\left(H^{d}\right)$ is the cohomology with respect to $\delta_{10 c}(d)$. 
Now we have the following theorem:

Theorem 5.1. Let $k<n, \phi_{p}^{k} \in S^{p, k}, d \phi_{p}^{k}=0$. Then $\exists \phi_{p}^{k-1} \in S^{p, k-1}$ such that $\phi_{p}^{k}=d \phi_{p}^{k-1}$. That is $H^{d}\left(S^{p, k}\right)=0$.

We postpone the proof until the end of the section.

From Theorem 5.1 and from the fact that $\delta_{\text {loc }} d=d \delta_{\text {loc }}$ one has:

$$
\delta_{1 \mathrm{oc}} \phi_{p}^{n}+\hat{d} \phi_{p+1}^{n-1}=0 \Rightarrow \exists \phi_{p+2}^{n-2} \quad \text { such that } \delta_{\mathrm{loc}} \phi_{p+1}^{n-1}+\hat{d} \phi_{p+2}^{n-2}=0,
$$

and so on all the way down to $\delta_{\text {loc }} \phi_{n+p}^{0}=0$. One has also:

$$
\phi_{p}^{n}=\delta_{\mathrm{loc}} \phi_{p-1}^{n}+\hat{d} \phi_{p}^{n-1} \Rightarrow \exists \phi_{p+1}^{n-2}
$$

such that:

$$
\phi_{p+1}^{n-1}=\delta_{\mathrm{loc}} \phi_{p}^{n-1}+\hat{d} \phi_{p+1}^{n-2},
$$

and so on all the way down to

$$
\phi_{p+n}^{0}=\delta_{1 \mathrm{oc}} \phi_{p+n-1}^{0} .
$$

So we have constructed an homomorphism

$$
H^{\delta_{1 \mathrm{oc}}} H^{d}\left(S^{p, n}\right) \rightarrow H^{\delta_{\mathrm{loc}}}\left(S^{p+n, 0}\right) .
$$

In other words, Theorem 5.1 says that the spectral sequence for the double complex $S$ is degenerated to the $E_{2}$ term (see [17]).

If also $H^{\delta_{10 c}}\left(S^{p^{\prime}, k}\right)$ is trivial for $k \geqq 1$, then (5.6) is an isomorphism; but generally this triviality condition is not satisfied.

Theorem 5.1 is also quoted in [10] and [16]. The homomorphism (5.6) is also in $[16]$.

Now for every ad-invariant multilinear symmetric map $f: \mathfrak{g} \times \ldots \times \mathfrak{g} \rightarrow \mathfrak{R}$ we can define in a natural way a map:

$$
\check{f}: \Omega^{i_{1}}(M ; \operatorname{ad} P) \times \Omega^{i_{2}}(M ; \operatorname{ad} P) \times \ldots \rightarrow \Omega^{i_{1}+i_{2}+\ldots}(M) .
$$

So if $K$ is the Killing form in $g$ and $/ 3$ is a trilinear symmetric invariant map, then $\psi_{3}^{0}$, defined by

$$
\psi_{3}^{0}\left(\xi_{1}, \xi_{2}, \xi_{3}\right)=\breve{K}\left(\xi_{1},\left[\xi_{2}, \xi_{3}\right]\right), \xi_{i} \in E
$$

and $\psi_{5}^{0}$ defined by

$$
\psi_{5}^{0}\left(\xi_{1}, \xi_{2}, \xi_{3}, \xi_{4}, \xi_{5}\right)=\check{f}_{3}\left(\xi_{1},\left[\xi_{2}, \xi_{3}\right],\left[\xi_{4}, \xi_{5}\right]\right), \xi_{i} \in E,
$$

are elements of $S^{3,0}$ and $S^{5,0}$, respectively. Moreover

$$
\psi_{3}^{0} \in H^{\delta_{10 c}}\left(S^{3,0}\right) \text { and } \psi_{5}^{0} \in H^{\delta_{100}}\left(S^{5,0}\right) .
$$

If $n=2, \psi_{3}^{0}$ is, up to a constant, the correspondent under the homomorphism (5.6) of the anomaly in two dimensions, $\psi_{1}^{2}$ (see e.g. [24]). If $n=4, \psi_{5}^{0}$ is, up to a constant, the correspondent under the homomorphism (5.6) of the Adler-BellJackiw anomaly $\psi_{1}^{4}[9,8]$. In local coordinates

$$
\psi_{1}^{2}(\xi)=\breve{K}(\xi, d \eta(A)), \xi \in E,
$$


and

$$
\begin{aligned}
\psi_{1}^{4}(\xi)= & \check{f}_{3}(\xi, d \eta(A), d \eta(A))+\frac{1}{4} \check{f}_{3}(\xi, d \eta(A),[\eta(A), \eta(A)]) \\
& -\frac{1}{2} \check{f}_{3}(\xi, \eta(A),[d \eta(A), \eta(A)]) \quad(\text { see }[16]) .
\end{aligned}
$$

These formulas take a more familiar aspect if we remember that when $P(M, G)$ is trivial we can choose $A_{0}=0$. The problem of the uniqueness of the anomaly is connected to the problem of inverting "somehow" the homomorphism (5.6); we will return to this problem in a forthcoming publication.

Proof of Theorem 5.1. The basic tool for proving Theorem 5.1 is a theorem by De Wilde [23].

Lemma 5.2 (De Wilde). Let $B$ be a vector bundle on $M, k<n$ and $L: \Gamma(B) \times \ldots \times \Gamma(B) \rightarrow \Omega^{k}(M)$ be a q-linear (symmetric, skew) map such that, $\forall \xi_{i} \in \Gamma(B), L\left(\xi_{1}, \ldots, \xi_{q}\right)$ is a closed form. Then there exists a q-linear (symmetric, skew) map $L^{\prime}: \Gamma(B) \times \ldots \times \Gamma(B) \rightarrow \Omega^{k-1}(M)$ such that $L=d L^{\prime}$.

Take now $k<n, \phi_{p}^{k} \in S^{p, k}$. If $\forall A$ and $\forall \xi_{i} \in E, \phi_{p}^{k}\left(\xi_{1}, \ldots, \xi_{p}\right)(A)$ is closed, then $\forall A \in \mathscr{A}, \exists \phi_{A}^{k-1, p}$ with:

$$
\phi_{A}^{k-1, p}: \underbrace{E \times \ldots \times E}_{p \text { times }} \rightarrow \Omega^{k-1}(M) \text { and } d \phi_{p}^{k-1}(A)=\phi_{p}^{k}(A), \forall A \in \mathscr{A} .
$$

We want to show that $\forall \xi_{i} \in E, \phi_{A}^{k-1, p}\left(\xi_{1}, \ldots, \xi_{p}\right) \in D^{k-1}\left(\mathscr{A}, A_{0}, M\right)$ or, equivalently, we want to prove that if $\psi \in D^{k}\left(\mathscr{A}, A_{0}, M\right)$ and $\psi(A)$ is exact $\forall A$, then $\exists \bar{\psi} \in D^{k-1}\left(\mathscr{A}, A_{0}, M\right)$ with $\psi(A)=d \bar{\psi}(A)$.

Indeed $\psi(A)=\sum_{m=0}^{s} L_{m}(\eta(A), \ldots, \eta(A))$, where $L_{m}$ are symmetric $m$-linear mappings from $\left(\Omega^{1}(M, \operatorname{ad} P)\right)^{m} \quad$ to $\quad \Omega^{k}(M)$. Now $d \psi(A)=0, \quad \forall A \in \mathscr{A}$ $\Rightarrow d L_{m}(\eta(A), \ldots, \eta(A))=0, \forall A \in \mathscr{A}$ and $\forall m \leqq s$. For any generic symmetric $m$-linear function $S$ of $n$ objects $a_{1}, a_{2}, \ldots, a_{m}$ belonging to a vector space, we can use the following combinatorial formula

$$
S\left(a_{1}, \ldots, a_{m}\right)=\frac{1}{m !} \sum_{k=0}^{m-1} \sum_{\substack{i_{1}, i_{2}, \ldots, i_{k}=1 \\ i_{1}<i_{2}<\ldots<i_{k}}}^{m}(-1)^{k} S\left(\sigma\left(a_{i_{1}}, \ldots, a_{i_{k}}\right), \ldots, \sigma\left(a_{i_{1}}, \ldots, a_{i_{k}}\right)\right),
$$

where $\sigma\left(a_{i_{1}}, \ldots, a_{i_{k}}\right)=a_{1}+\ldots+\hat{a}_{i_{1}}+\ldots+\hat{a}_{i_{k}}+\ldots+a_{m}$ and the symbol ^ means that the corresponding term is missing in the sum. Let us apply this formula to $L_{m}$

$$
L_{m}\left(\eta_{1}, \ldots, \eta_{m}\right)=\sum_{j} c_{j} L_{m}\left(\bar{\eta}_{j}, \ldots, \bar{\eta}_{j}\right),
$$

where $c_{j}$ are suitable coefficients and the arguments of $L_{m}$ in the right hand side are all equal. Then

$$
d L_{m}(\eta(A), \ldots, \eta(A))=0, \forall A \Rightarrow d L_{m}=0,
$$

and by Lemma 5.2 again $\exists L_{m}^{\prime}$ such that $L_{m}=d L_{m}^{\prime}$. Finally

$$
\bar{\psi}(A)=\sum_{m=0}^{s} L_{m}^{\prime}(\eta(A), \ldots, \eta(A))
$$




\section{Some Comments}

We think that the local cohomology introduced in Sect. 5 is a relevant object for applications in gauge theories. Solving this cohomology or proving that the relative cohomology spaces are finite dimensional seems to imply some mathematical hardship.

As for the geometrical interpretation of ghosts and BRS transformations, we observe that apparently the shades of Thierry-Mieg's scheme ([6] and also [7]) are absent here. As it should be, the ghosts depend directly on the base space variables, anti-ghosts and anti-BRS transformations are explained in a natural way; one can construct non-trivial functionals with an arbitrary number of ghosts. (While this work was in progress we saw a paper [25] which also suggests that ghosts may be related to the Maurer-Cartan form on the group of gauge transformations.) Matter fields could easily fit in the scheme of this paper: they are sections of vector bundles associated to $P(M, G)$ and the group $\mathscr{G}$ acts on these sections, so we can extend to them the operations we have been constructing here.

In conclusion, the geometrical objects and operations introduced in this paper seem to parallel exactly ghost fields and BRS transformations in field theory.

There seems also to be a connection between the superfield formalism for gauge theories (see [5] and recently [24]) and the present paper. The Grassman algebra underlying the superfield formalism is likely to be the exterior algebra over $E^{*}$ and the superfield formalism itself an operative device for dealing with it.

\section{Appendix: Anti-BRS Transformations}

In this appendix, we want to give a geometrical interpretation of the anti-BRS transformations. The anti-BRS transformations, denoted by $\bar{\jmath}$, are usually written as :

$$
\begin{aligned}
& A_{\mu}^{a}(x) \rightarrow A_{\mu}^{a}(x)+\bar{\lambda} \bar{s}\left(A_{\mu}^{a}(x)\right)=A_{\mu}^{a}(x)+\bar{\lambda}\left(D_{\mu} \bar{c}(x)\right)^{a}, \\
& \bar{c}^{a}(x) \rightarrow \bar{c}^{a}(x)+\bar{\lambda} \bar{J}\left(\bar{c}^{a}(x)\right)=\bar{c}^{a}(x)-\frac{1}{2} \bar{\lambda} f^{a b d} \bar{c}^{b}(x) \bar{c}^{d}(x), \\
& c^{a}(x) \rightarrow c^{a}(x)+\bar{\lambda} \bar{J}\left(c^{a}(x)\right)=c^{a}(x)+\bar{\lambda} \bar{B}^{a}(x),
\end{aligned}
$$

where

$$
B^{a}(x)+\bar{B}^{a}(x)+f^{a b d} \bar{c}^{b}(x) c^{d}(x)=0,
$$

$\bar{\lambda}$ is an anticommuting parameter and the other notations are the same as in (2.1). Of course an equation like (2.2) holds also for $\bar{c}(x)$, and so $\bar{\jmath}^{2}=0$. Moreover due to (A2) one has:

$$
\begin{aligned}
(\jmath+\bar{J})\left[D_{\mu} c(x)+D_{\mu} \bar{c}(x)\right]^{a}= & -\frac{1}{2}\left(D_{\mu}[c(x), c(x)]\right)^{a} \\
& +f^{a b d}\left(D_{\mu} c(x)\right)^{b} c^{d}(x)-\frac{1}{2}\left(D_{\mu}[\bar{c}(x), \bar{c}(x)]\right)^{a} \\
& +f^{a b d}\left(D_{\mu} \bar{c}(x)\right)^{b} \bar{c}^{d}(x)+f^{a b d}\left(D_{\mu} c(x)\right)^{b} \bar{c}(x) \\
& +f^{a b d}\left(D_{\mu} \bar{c}(x)\right)^{b} c^{d}(x)+\left(D_{\mu}(B(x)+\bar{B}(x))\right)^{a}=0,
\end{aligned}
$$

that is, $(s+\bar{J})^{2}=5 \bar{s}+\overline{5} s=0$. 
Let's now take $\mathscr{G}^{\prime}=\mathscr{G}^{\prime \prime}=\mathscr{G}$ and let $\omega^{\prime}$ and $\omega^{\prime \prime}$ be the Maurer-Cartan forms in $\mathscr{G}^{\prime}$ and $\mathscr{G}^{\prime \prime}$, respectively. Here $\omega^{\prime}$ is the ghost field and $\omega^{\prime \prime}$ is the antighost field. Then $E^{\prime}$ and $E^{\prime \prime}$ will be the Lie algebra of $\mathscr{G}^{\prime}$ and $\mathscr{G}^{\prime \prime}$, respectively.

For each connection $A$ and for each $\xi^{\prime} \in E^{\prime}, \xi^{\prime \prime} \in E^{\prime \prime}, A+d_{A} \omega^{\prime}\left(\xi^{\prime}\right)+d_{A} \omega^{\prime \prime}\left(\xi^{\prime \prime}\right)$ is another connection, $d_{A} \omega^{\prime}+d_{A} \omega^{\prime \prime}$ is a $\Omega^{1}(M$, ad $P)$-valued 1 -form on the direct product $\mathscr{G}^{\prime} \times \mathscr{G}^{\prime \prime}$ and in particular is the derivative at $g^{\prime}=g^{\prime \prime}=$ identity, of the map $\chi_{A}: \mathscr{G}^{\prime} \times \mathscr{G}^{\prime \prime} \rightarrow \mathscr{A}$ given by $\chi_{A}\left(g^{\prime}, g^{\prime \prime}\right)=\left(A \cdot g^{\prime}\right) \cdot g^{\prime \prime}$, if $g^{\prime} \in \mathscr{G}^{\prime}, g^{\prime \prime} \in \mathscr{G}^{\prime \prime}$, and $A \in \mathscr{A}$. The transformation

$$
A \rightarrow A+d_{A} \omega^{\prime}+d_{A} \omega^{\prime \prime}
$$

stands for

$$
\left(A, \xi^{\prime}, \xi^{\prime \prime}\right) \rightarrow A+d_{A} \omega^{\prime}\left(\xi^{\prime}\right)+d_{A} \omega^{\prime \prime}\left(\xi^{\prime \prime}\right), \quad\left(\xi^{\prime}, \xi^{\prime \prime}\right) \in E^{\prime} \times E^{\prime \prime}
$$

and corresponds to the transformation $A \rightarrow A+(\jmath+\bar{J})(A)$.

Let's apply twice the transformation $\left(\mathrm{A} 4^{\prime}\right)$ antisymmetrizing with respect to the vector fields $\xi^{\prime}, \zeta^{\prime} \in E^{\prime}$ and separately to the vector fields $\xi^{\prime \prime}, \zeta^{\prime \prime} \in E^{\prime \prime}$. We have

$$
\begin{aligned}
A & \rightarrow A+d_{A}\left[\omega^{\prime}\left(\xi^{\prime}\right), \omega^{\prime}\left(\zeta^{\prime}\right)\right]+d_{A}\left[\omega^{\prime \prime}\left(\xi^{\prime \prime}\right), \omega^{\prime \prime}\left(\zeta^{\prime \prime}\right)\right] \\
& +d_{A}\left[\omega^{\prime \prime}\left(\xi^{\prime \prime}\right), \omega^{\prime}\left(\zeta^{\prime}\right)\right]+d_{A}\left[\omega^{\prime}\left(\xi^{\prime}\right), \omega^{\prime \prime}\left(\zeta^{\prime \prime}\right)\right] .
\end{aligned}
$$

Let us define now for practical convenience two $E$-valued 1 -forms on $\mathscr{G}_{1} \times \mathscr{G}_{2}$, $\omega_{1}$ and $\omega_{2}$ :

$$
\omega_{1}\left(\begin{array}{l}
\xi^{\prime} \\
\xi^{\prime \prime}
\end{array}\right)=: \omega^{\prime}\left(\xi^{\prime}\right) ; \quad \omega_{2}\left(\begin{array}{l}
\xi^{\prime} \\
\xi
\end{array}\right)=: \omega^{\prime \prime}\left(\xi^{\prime \prime}\right) .
$$

Of course we can thaink of $\omega_{1}$ and $\omega_{2}$ also as the ghost and the antighost. We extended the definition (3.5) for forms on $\mathscr{G}_{1} \times \mathscr{G}_{2}$ and have:

$$
\left[d_{A}\left(\omega_{1}+\omega_{2}\right), \omega_{1}+\omega_{2}\right]-\frac{1}{2} d_{A}\left[\omega_{1}, \omega_{1}\right]-\frac{1}{2} d_{A}\left[\omega_{2}, \omega_{2}\right]-d_{A}\left[\omega_{1}, \omega_{2}\right]=0,
$$

which is the same as Eq. (A3) with condition (A2). Equation (A6) implies that if we add to the result of the transformation (A5) the term

$$
\left(-\frac{1}{2} d_{A}\left[\omega_{1}, \omega_{1}\right]-\frac{1}{2} d_{A}\left[\omega_{2}, \omega_{2}\right]-d_{A}\left[\omega_{1}, \omega_{2}\right]\right)\left(\left(\begin{array}{c}
\xi^{\prime} \\
\zeta^{\prime}
\end{array}\right),\left(\begin{array}{c}
\xi^{\prime} \\
\zeta^{\prime \prime}
\end{array}\right)\right)
$$

we get to the connection $A$, where we have started from; this corresponds to the nilpotency of the sum of BRS and anti-BRS transformations. Notice that the geometric structure of the BRS-anti-BRS transformations does not involve the auxiliary fields $B^{a}, \bar{B}^{a}$.

Acknowledgements. Discussions with Raoul Bott have been particularly enlightening and we thank him warmly for that. Useful discussions with J. Thierry-Mieg, D. Groisser, and T. Parker are also acknowledged. Moreover P. C.-R. wants to thank Arthur Jaffe for the hospitality at Harvard; L. B. wishes to do the same with the Center for Theoretical Physics at M.I.T. 


\section{References}

1. Becchi, C., Rouet, A., Stora, R. : Renormalization of gauge theories. Ann. Phys. 98, 287 (1976)

2. Becchi, C., Rouet, A., Stora, R.: Renormalization of the abelian Higgs-Kibble model. Commun. Math. Phys. 42, 127 (1975)

3. Curci, G., Ferrari, R.: Slavnov transformations and supersymmetry. Phys. Lett. 63B, 91 (1976)

4. Kugo, T., Ojima, I.: Local covariant operator formalism of non abelian gauge theories and quark confinement problem. Prog. Theor. Phys. Suppl. 66, (1979)

5. Bonora, L., Tonin, M.: Superfield formulation of extended BRS symmetry. Phys. Lett. 96B, 48 (1981)

6. Thierry-Mieg, J.: Geometrical reinterpretation of Faddeev-Popov ghost particles and BRS transformation. J. Math. Phys. 21, 2834 (1980)

7. Quirós, M., de Urries, I.S., Hoyos, J., Mazon, M.L., Rodrigues, E.: Geometrical structure of Faddeev-Popov fields and invariance properties of gauge theories. J. Math. Phys. 22, 1767 (1981)

8. Bell, J.S., Jackiw, R.: A PCAC puzzle: $\pi^{0} \rightarrow \gamma \gamma$ in the $\sigma$-model. Nuovo Cimento A60, 47 (1969)

9. Adler, S.L. : Axial-vector vertex in spinor electrodynamics. Phys. Rev. 177, 2426 (1969)

10. Dixon, J.A.: Cohomology and renormalization of gauge theories. I-III (unpublished)

11. Atiyah, M., Bott, R.: On the Yang-Mills equations over Riemann surfaces (preprint)

12. Singer, I.M.: Some remarks on the Gribov ambiguity. Commun. Math. Phys. 60, 7 (1978)

13. Narasimhan, M.S., Ramadas, T.R.: Geometry of SU(2) gauge fields. Commun. Math. Phys. 67, 121 (1979)

14. Mitter, P.K., Viallet, C.M.: On the bundle of connections and the gauge orbit manifold in YangMills theory. Commun. Math. Phys. 79, 457 (1981)

15. Mitter, P.K.: In: Recent developments in gauge theories. t'Hooft, G. et al. (eds.). New York, London: Plenum Press 1980

16. Stora, R.: In: New developments in quantum field theories and statistical mechanics. Levy, H., Mitter, P. (eds.). New York, London: Plenum Press 1977

17. Bott, R., Tu: Differential forms in algebraic topology. Berlin, Heidelberg, New York: Springer 1982

18. Georgi, H., Glashow, S.L.: Gauge theories without anomalies. Phys. Rev. D6, 429 (1972)

19. Okubo, S.: Gauge groups without triangular anomaly. Phys. Rev. D16, 3528 (1977)

20. Groisser, D.: Unpublished

21. Greub, W., Halperin, S., Vanstone, R.: Connections, curvature, and cohomology, Vol. III. New York: Academic Press 1976

22. Peetre, J.: Une caracterisation abstraite des operateurs differentiels. Math. Scand. 7, 211 (1959)

23. De Wilde, M.: On the local Chevalley cohomology of the dynamical Lie algebra of a symplectic manifold. Lett. Math. Phys. 5, 351 (1981)

24. Bonora, L., Cotta-Ramusino, P.: ABJ anomalies and superfield formalism in gauge theories. Phys. Lett. B 107, 87 (1981)

25. Leinaas, J.M., Olaussen, K.: Ghosts and geometry. CERN Preprint TH. 3061 (1981)

Communicated by R. Stora

Received February 5, 1982; in revised form September 7, 1982 
